\section{Renal Disease in Pregnancy}

\author{
John M Davison, Catherine Nelson-Piercy, Sean Kehoe \\ and Philip Baker \\ ISBN: 978-1-904752-59-2 \\ London: RCOG Press, 2008 \\ $£ 80$
}

Renal disease in pregnancy has historically been an area of controversy and discomfort for many nephrologists and obstetricians. Women with renal disease are clearly at increased risk of fetal and maternal complications and, in the past, were dissuaded from attempting to become pregnant. However, attitudes have changed and chronic kidney disease (CKD) is no longer considered an absolute contraindication to pregnancy. Today, pregnancy in the setting of renal disease is becoming more common. As mentioned in Chapter 2, between 3\% and $10 \%$ of women of child-bearing age might be defined as having CKD when using the estimation of glomerular filtration rate rather than serum creatinine to assess renal function. ${ }^{1}$

Renal Disease in Pregnancy, which stems from the 54th RCOG Study Group on 'Renal Disease in Pregnancy', is a timely and much-needed comprehensive review of clinical practice within the field. The book is divided into eight sections. The first section gives a basic review of renal physiology in pregnancy and a simple explanation of angiogenic factors. This level of explanation is geared towards the general nephrologist or obstetrician, which is ideal for such a book. This portion of the book includes a section on the potential role of midwifery in the very high-risk patient, which may prove feasible and appealing in some high-risk centres. The second section deals with the general principles of prepregnancy counselling, management during pregnancy and postpartum care. Given the complexity of issues and the paucity of high-quality studies in this area, the authors have done an excellent job of outlining and estimating risk in ways that physicians can easily convey to their patients. They discuss the controversy and paucity of data, but still draw reasonable conclusions from the available information - something rarely done in this area.

The third section is divided into chapters on specific areas within CKD. This section does an excellent job of summarizing dialysis, renal transplantation and diabetic nephropathy. However, the chapter on lupus and pregnancy might be considered too general for most nephrologists and rheumatologists who specialize in this condition. The last four sections cover drugs used in renal disease, acute renal impairment with a very thoughtful approach to kidney biopsy in the pregnant patient, urologic issues and issues specific to renal transplant. The chapter on assisted reproduction was interesting, given that the topic is usually excluded from most articles or books dealing with renal disease. The final section is a concise summary of the consensus views from the conference and future research goals, which were discussed throughout the book.

One criticism of the book is that non-peer-reviewed and unpublished data are referred to and one wonders whether these data have the equivalent scientific rigour of published data. Having said this, some of the data are fascinating and compelling (e.g. a registry of outcomes from 908 pregnancies in 676 women with renal disease) and one regrets that it has not been published and available to aid in clinical decision making.

Overall, the book is well researched, organized and easy to read, and summarizes the literature and evidence to date. Renal Disease in Pregnancy is a much needed addition to the literature and an excellent resource for general nephrologists, high-risk obstetricians, trainees and all those involved in the medical care of this special population of pregnant women.

\section{Geena Joseph and Michelle Hladunewich}

Department of Medicine, University of Toronto, Toronto, Canada Email: michelle.hladunewich@sunnybrook.ca DOI: 10.1258/om.2009.090016

\section{REFERENCE}

1 E Imbasciati, G Gregoriniand G Cabidduet al. Pregnancy in CKD stages 3 to 5: fetal and maternal outcomes. Am J Kid Dis 2007;49:753-62

\section{Heart Disease in Pregnancy, 2nd Edition}

\author{
Celia Oakley and Carole A. Warnes \\ ISBN 978-1-4051-3488-0
}

Oxford: BMJ Books, Wiley-Blackwell, 2007

$£ 72.99$

This is the second edition of a comprehensive textbook originally published in 1997. Its scope is wide and includes useful chapters on subjects such as cor pulmonale and hypertensive disorders of pregnancy, which are highly relevant but outside the practice of most cardiologists. There are also excellent sections on contraception and genetic counselling, reinforcing the important message that good pregnancy care begins preconception.

The book displays both the advantages and disadvantages of a multiauthor text. The reader benefits from the experience of many experts but there is significant, often inconsistent, overlap between chapters. Further editing and cross-referencing would improve this greatly. While many chapters are excellent, giving a clear summary of the evidence and current best practice, others are out of date even allowing for the time lag of publication. In addition, several tables and diagnostic strategy diagrams contain typographical errors major enough to render them useless. Authors also vary in their approach between qualitative overview and practical management with clear parameters. In my experience, readers value as much of the latter as possible.

Heart Disease in Pregnancy appropriately emphasizes the importance of multidisciplinary care and would be of interest 